\title{
Orthodontic retention: a final frontier?
}

\author{
Padhraig S. Fleming ${ }^{1}$ and Simon J. Littlewood ${ }^{2}$ \\ ${ }^{1}$ Professor of Orthodontics, Queen Mary University of London; ${ }^{2}$ Consultant Orthodontist, Bradford Teaching Hospitals \\ NHS Foundation Trust, UK
}

The BDJ Upfront section includes editorials, letters, news, book reviews and interviews. Please direct your correspondence to the News Editor,

Kate Quinlan at k.quinlan@nature.com. Press releases or articles may be edited, and should include a colour photograph if possible.

$\mathrm{T}$ he phrase 'Retention is the final frontier' in orthodontics has been mooted. For experienced practitioners and those immersed in both the theory and practice of clinical orthodontics, this may appear somewhat trite. Orthodontics presents many challenges and frontiers involving a complex interplay between an array of considerations including, but not confined to, patient concerns and motives, skeletal and soft tissue relationships, tooth size and shape, and periodontal phenotype. As such, problems and quandaries are common, and binary decisions are the exception rather than the rule.

Clearly, however, retention continues to be a burning issue. This is entirely intuitive given the incongruous aim of obtaining 'artificial stability' by both mitigating the possibility of direct relapse following active tooth movement and even halting physiological, age-related maturation. The latter, in particular, presents an enigma dictating indefinite retention to conceal the inevitable effects of ageing. The openended nature of the post-orthodontic period introduces clear challenges in terms of the effectiveness and safety of the approach to retention. The pervasion of orthodontics in all its forms among both adolescents and adults, allied to the challenges of undertaking regular post-treatment review by specialist providers, ensures that a deep appreciation of the rationale for retention and contemporary approaches to both fixed and removable approaches is important among both orthodontists and dentists.

Orthodontics has witnessed significant change in recent decades with advancement in appliance design and a growing appreciation of the centrality of patient experiences and outcomes. Planning around a putative stable mandibular incisor position has diminished somewhat. This paradigm shift has been mirrored by a tacit acknowledgement that retention is an imperative should complete stability be targeted. Crowding is also increasingly prevalent and exacerbated by the effects of growth and maturation of hard and soft tissues. Within this 'perfect storm', producing ideal orthodontic outcomes in the long-term remains an enduring dilemma. need for retention, and can understand and accept their central role in successful retention, irrespective of the mechanical approach used. Moreover, there may well be varying tolerance for post-treatment change. Based on a growing body of evidence, there is also an increasing appreciation of the relative stability of a range of intra- and inter-arch features, allied to the associated need for bespoke approaches to retention. Again, an understanding of this is helpful in planning active treatment, informing treatment decisions and managing patient expectations.

\section{'It is fundamental that patients appreciate the implications of orthodontic treatment and the subsequent need for retention'}

In this issue we unpick a number of these aspects in what we hope is a relatively complete guide to orthodontic retention offering equal value both to orthodontists, orthodontic trainees, non-specialist providers and general dentists. In particular, we detail the accepted mechanical means of affecting retention; namely, fixed and removable approaches with presentation of theoretical underpinning and practical guides. There are peculiar challenges associated with the longterm, indeed indefinite, nature of retention, and the implications of these mechanical approaches for patients and supervising clinicians. As such, we attempt a holistic overview of the effects of retention on both patients and providers. It is fundamental that patients appreciate the implications of orthodontic treatment and the subsequent
Retention does undoubtedly remain a frontier and indeed a conundrum. Teeth are pre-determined to move throughout life. This physiological change may be acceptable to many but anathema to others. Tailored solutions are therefore currently predicated on mechanical approaches with patient understanding and motives necessarily central to this.

We are deeply indebted to each of the contributors for their invaluable contributions, and hope that we have collectively provided deeper context and demystified the many challenges associated with retention. Alas, we are unlikely to have breached this stubborn frontier, but we very much hope that you will enjoy reading the articles in this themed issue.

https://doi.org/10.1038/s41415-021-3017-9 